\title{
ATF2 translation is induced under chemotherapeutic drug-mediated cellular stress via an IRES-dependent mechanism in human hepatic cancer Bel7402 cells
}

\author{
QI LI* ${ }^{*}$ WEN-QING GAO*, WEN-YAN DAI*, CHUANG YU, RUI-YU ZHU and JIAN JIN \\ Laboratory of Molecular Pharmacology, School of Pharmaceutical Sciences, \\ Jiangnan University, Wuxi, Jiangsu 214122, P.R. China
}

Received April 17, 2015; Accepted August 9, 2016

DOI: $10.3892 / 01.2016 .5274$

\begin{abstract}
Activating transcription factor (ATF) 2 is a member of the ATF/cyclic AMP-responsive element binding protein family, which exhibits both oncogenic and tumor-suppressor functions. In our preliminary experiments, it was observed that the expression of the ATF2 protein was induced following treatment with adriamycin (ADR) and paclitaxel (PTX), which may be regulated by internal ribosome entry segment (IRES)-mediated translation. By constructing a bicistronic vector containing the ATF2 5'-untranslated region (UTR), it was demonstrated that the ATF2 5'-UTR contains an IRES and maps a 30-nucleotide (nt) sequence (from nt 299 to $\mathrm{nt}$ 269), which was essential for the IRES activity. The ATF2 IRES activity exhibited significant variation in different cell lines. In addition, it was observed that ADR and PTX also induced ATF2 IRES activity in Bel7402 cells. The present study has demonstrated that ATF2 translation is initiated via IRES, which is upregulated by ADR and PTX, thus suggesting that the regulation of the IRES-dependent translation of ATF2 may be involved in effecting the cancer cell response to chemotherapeutic drugs-mediated cellular stress.
\end{abstract}

\section{Introduction}

Activating transcription factor (ATF) 2 is a member of the ATF/cyclic AMP-responsive element binding protein family of transcription factors, which harbors leucine zipper and

Correspondence to: Professor Jian Jin or Dr Ruiyu Zhu, Laboratory of Molecular Pharmacology, School of Pharmaceutical Sciences, Jiangnan University, 1800 Lihu Road, Wuxi, Jiangsu 214122, P.R. China

E-mail: jinjian31@126.com

E-mail: ry_zhu@sina.com

${ }^{*}$ Contributed equally

Key words: internal ribosome entry site, activating transcription factor 2, cellular stress, translation
DNA binding domains (1), and exhibits both oncogenic and tumor-suppressor functions (2-4). ATF2 is important for normal cellular development and survival, and is also involved in the response to stress and DNA damage in the context of normal and tumorigenesis processes (5). The transactivating potential of ATF2 is stimulated to a higher level by a broad group of agents causing DNA damage and other types of cellular stress, including short-wavelength ultraviolet (UV) light, osmotic stress and inflammatory cytokines (6,7). ATF2 is expressed ubiquitously, and elevated expression of ATF2 was detected in skin carcinomas (8) and melanoma (9). It was reported that the ATF2 expression levels were significantly induced under oxidative stress in immortalized human urothelial cells treated with arsenic (10).

Translational control is important in the control of gene expression, by which, cells can respond to rapid changes in physiological conditions, including DNA damage and cellular stress $(11,12)$. One of the common mechanisms of translational control is the induction of changes in the translation initiation site on the messenger RNA (mRNA) molecule, which may occur via two distinct mechanisms, cap-dependent scanning and internal ribosome entry (IRE) (13). The latter mechanism requires the formation of a complex RNA structural element termed IRE segment (IRES), which is located in the 5'-untranslated region (UTR) of the mRNA molecule (14). To date, IRES elements have been mainly identified in mRNAs involved in regulating gene expression during development, differentiation, cell cycle progression, cell growth, apoptosis and stress, including nuclear factor- $\kappa \mathrm{B}$ repressing factor, $\mathrm{X}$-chromosome linked inhibitor of apoptosis protein (XIAP), vascular endothelial growth factor, c-Myc, fibroblast growth factor-2 and Smad mRNAs (15-19). The most notable difference in cellular IRES sequence from the UTR data sets is that IRES-containing UTRs are generally long ( $>150$ bases) and GC rich (20).

Multiple cellular stresses lead to the inhibition of translation; however, there are certain cellular mRNAs that are selectively translated under these conditions to protect cells from stress, the majority of which contain an IRES element (21). Yang et al noticed that tumor necrosis factor receptor-associated factor 1 protein expression can be stimulated under cellular stress conditions, and its translation is regulated by IRES (22). In addition, it was reported that ATF4 splice variant, whose 
protein expression was induced by thapsigargin and tunicamycin in THP-1 cells, contains an IRES structure (23).

Adriamycin (ADM) and paclitaxel (PTX) are two effective antitumor agents that are widely used in hepatocellular carcinoma therapy $(24,25)$. Both can induce cell apoptosis and cause cellular stress (26). In the present study, ATF2 protein expression in the human hepatoma cell line Bel7402 was observed to be induced following treatment with ADM or PTX, and it was demonstrated that the 5'-UTR of ATF harbors a bona fide IRES structure localized between nt -299 to nt -269 upstream of the start codon. The IRES activity of ATF2 was low in the mouse embryo fibroblast cell line National Institutes of Health (NIH)-3T3 and in the human colon carcinoma cell line HCT- 8 , while it was high in the human embryonic kidney (HEK) 293 cell line and in the human hepatoma cell line Bel7402. Furthermore, the present study was demonstrated that ATF2 IRES activity was also elevated following treatment with cellular stress-inducing chemotherapeutic drugs such as PTX and ADM.

\section{Materials and methods}

Plasmid construction. The bicistronic vector pRF was constructed by inserting the firefly luciferase (FL or FLUC) gene from the plasmid pGL-6 (Beyotime Institute of Biotechnology, Haimen, China) into the linker region of the plasmid pRL-SV4 (Promega Corporation, Madison, WI, USA) downstream of the Renilla luciferase (RL or RLUC) gene. To insert the FLUC fragment, a polymerase chain reaction (PCR) was performed on the pGL-6 plasmid with primers FLUCF 5'-GCT CTAGACTCGAGCATATGGAATTCGGCGCGCCCATGG AAGATGCCAAAAACATTAAGAAGGGCCC-3' (forward) and FLUCR 5'-AAATATGCGGCCGCTTACACGGCGATC TTGCCGCCCTT-3' (reverse). The PCR was conducted in a 50- $\mu 1$ reaction mixture containing $2 \mathrm{X}$ PrimeSTAR Max Premix (Takara Biotechnology Co., Ltd., Dalian, China), $0.2 \mu \mathrm{M}$ forward and reverse primers, and $200 \mathrm{ng}$ genomic DNA, which was purchased from the Beyotime Institute of Biotechnology. The PCR was conducted at $98^{\circ} \mathrm{C}$ for $3 \mathrm{~min}$, followed by 32 cycles of $98^{\circ} \mathrm{C}$ for $1 \mathrm{~min}, 55^{\circ} \mathrm{C}$ for $10 \mathrm{sec}$ and $72^{\circ} \mathrm{C}$ for $1 \mathrm{~min}$, and $72^{\circ} \mathrm{C}$ for $5 \mathrm{~min}$. The amplified product was digested with $B s p 119$ and $\mathrm{XbaI}$, and subsequently cloned into pRL-SV40, thus generating pRF. In this vector, the two cistrons were separated by $\sim 20$ base pairs (bp) of the intercistronic linker region containing several unique endonuclease restriction sites. The expression of bicistronic mRNA is driven by a simian virus (SV) 40 promoter. The full length 5'-UTR complementary DNA (cDNA) of ATF2, nuclear respiratory factor (NRF) and XIAP were obtained by gene synthesis (Sangon Biotech Co., Ltd., Shanghai, China), while serial truncations of ATF2 were produced by PCR amplification with forward and reverse primers (Table I) containing EcoRI and $N d e I$ endonuclease restriction sites, respectively. The PCR was conducted in a 50- $\mu 1$ reaction mixture containing 2X PrimeSTAR Max Premix, 0.2 $\mu \mathrm{M}$ forward and reverse primers, and $200 \mathrm{ng}$ genomic DNA. The PCR was conducted at $98^{\circ} \mathrm{C}$ for $3 \mathrm{~min}$, followed by 32 cycles of $98^{\circ} \mathrm{C}$ for $1 \mathrm{~min}$, varying annealing temperatures (as indicated in Table I) and $72^{\circ} \mathrm{C}$ for $10 \mathrm{sec}$, and $72^{\circ} \mathrm{C}$ for $5 \mathrm{~min}$. All these $5^{\prime}$-UTR cDNAs were each inserted immediately upstream of the translation start codon of the FLUC gene in the dual luciferase vector $\mathrm{pRF}$ at the EcoRI and NdeI sites.

Cell culture and DNA transfection. The HEK293 cell line (Type Culture Collection of the Chinese Academy of Sciences, Shanghai, China) was cultured in Dulbecco's modified Eagle medium (Gibco; Thermo Fisher Scientific, Inc., Waltham, MA, USA), while the Bel7402, HCT-8 and NIH-3T3 cell lines (Type Culture Collection of the Chinese Academy of Sciences) were cultured in RPMI 1640 medium (Gibco; Thermo Fisher Scientific, Inc.) with $10 \%$ (vol/vol) fetal bovine serum (Gibco; Thermo Fisher Scientific, Inc.), penicillin (100 U/ml) and streptomycin $(100 \mu \mathrm{g} / \mathrm{ml})$. All cells were kept at $37^{\circ} \mathrm{C}$ in a humidified atmosphere with $5 \% \mathrm{CO}_{2}$. Transient transfection was performed using Lipofectamine 2000 (Invitrogen; Thermo Fisher Scientific, Inc.)according to the manufacturer's protocol. The cell media were replaced with complete culture media at $6 \mathrm{~h}$ post-transfection, and 24-48 h later, the cells were rinsed twice with phosphate-buffered saline, and cell extracts were then prepared using 5X Passive Lysis Buffer (Promega Corporation).

Dual luciferase assay. FLUC and RLUC activities were measured using the Dual-Luciferase Reporter Assay System (Promega Corporation) according to the manufacturer's protocol, with the exception that only $100 \mu \mathrm{l}$ of each reagent was used. The signals were measured with a luminometer.

$R N A$ isolation and reverse transcription $(R T)-P C R$. Total RNA was isolated from the cells using TRIzol reagent (Beyotime Institute of Biotechnology), and was reverse transcribed into cDNA using oligo(dT)18 primer (Takara Biotechnology Co., Ltd.) and PrimeScript Reverse Transcriptase (Takara Biotechnology Co., Ltd.). PCR was next performed using specific primers, which were designed according to the corresponding sequences of the ATF2 5'-UTR mRNA: ATF2F 5'-GCACGT AATGACAGTGTCATTGT-3' (forward) and ATF2R 5'-ATG TGGGCTGTGCAGTTTGTG-3' (reverse). The PCR was conducted in a 50- $\mu 1$ reaction mixture containing $2 \mathrm{X}$ PrimeSTAR Max Premix, $0.2 \mu \mathrm{M}$ forward and reverse primers, and $250 \mathrm{ng}$ total RNA. The PCR was conducted at $98^{\circ} \mathrm{C}$ for $3 \mathrm{~min}$, followed by 32 cycles of $98^{\circ} \mathrm{C}$ for $1 \mathrm{~min}, 51.6^{\circ} \mathrm{C}$ for $15 \mathrm{sec}$ and $72^{\circ} \mathrm{C}$ for $18 \mathrm{sec}$, and $72^{\circ} \mathrm{C}$ for $5 \mathrm{~min}$. Upon electrophoresis on a $1.5 \%$ agarose gel, the PCR products were visualized with ethidium bromide staining under UV light. DNA bands were analyzed using ImageJ software (NIH, Bethesda, MD, USA).

Western blotting. Whole-cell proteins were obtained using radioimmunoprecipitation assay buffer containing $1 \mathrm{mM}$ phenylmethane sulfonyl fluoride. The same quantity of total protein was loaded onto each lane of the gel, and the proteins were electrophoresed on $8 \%$ polyacrylamide gels containing $0.1 \%$ sodium dodecyl sulfate. The resolved proteins were semi-dry transferred to a polyvinylidene fluoride membrane. The membrane was blocked with $0.5 \%$ bovine serum albumin (Beyotime Institute of Biotechnology) at room temperature for $\sim 3 \mathrm{~h}$, prior to be incubated with the corresponding primary antibodies [anti-ATF2 (1:5,000; ab32061; Abcam, Cambridge, MA, USA) or anti- $\beta$-actin (1:1,000; AA128; Beyotime Institute of Biotechnology), which was used as an internal reference] 
Table I. Oligonucleotide primers used in polymerase chain reaction for plasmids construction.

\begin{tabular}{llc}
\hline Vector (nt) & \multicolumn{1}{c}{ Primer sequence, 5'-3' } & Temperature, ${ }^{\circ} \mathrm{C}$ \\
\hline pR-ATF2-F (31-299) & F1: GGAATTCCATATGGAAGCCTGTGGGAGCCC & 50.0 \\
& R1: CGGAATTCAAGTTGAATAACTTATCACATTC & \\
pR-ATF2-F (51-299) & F2:GGAATTCATATGGCCTTTAAAGTGCCGTTCAG & 50.0 \\
& R2: CGGAATTCAAGTTGAATAACTTATCACATTC & \\
pR-ATF2-F (76-299) & F3: GGAATTCCATATGTCCTCCAGGGGTGCTTTGTA & 50.0 \\
& R3: CGGAATTCAAGTTGAATAACTTATCACATTC & 50.0 \\
pR-ATF2-F (141-299) & F4: GGAATTCCATATGACTAGTGACCTGGAAAGGGT & 52.0 \\
pR-ATF2-F (1-229) & R4: CGGAATTCAAGTTGAATAACTTATCACATTC & 52.8 \\
FR-ATF2-F (1-159) & F5: GGAATTCCATATGGTTGTCAGTCCGATCTCGC & 5 \\
pR-ATF2-F (1-89) & F6: GGAATTCCATATGGTTGTCAGTCCGATCTCGC & 54.5 \\
& R6: CGGAATTCCCCTTTCCAGGTCACTAGTTC & \\
\hline
\end{tabular}

F, forward; R, reverse; ATF2, activating transcription factor 2.

A

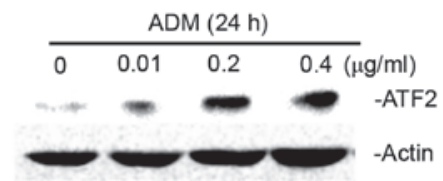

B

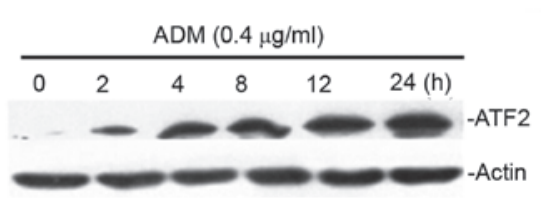

C

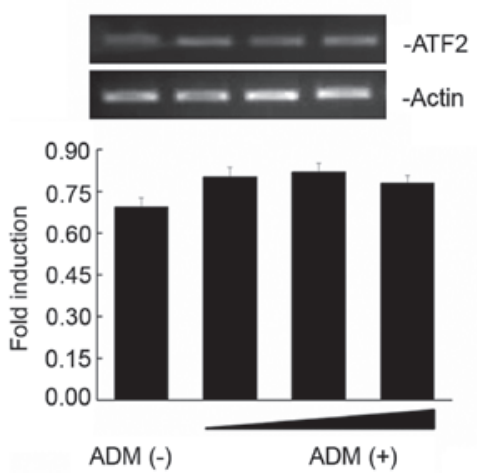

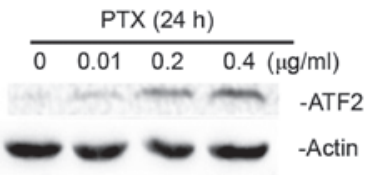
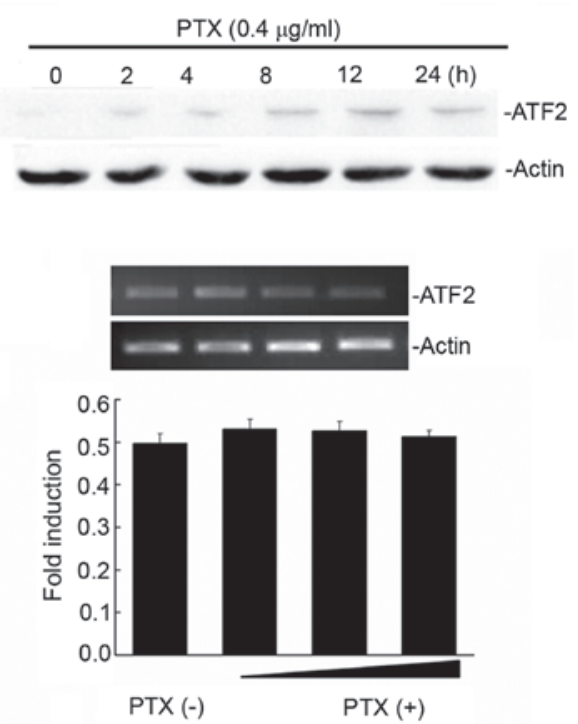

Figure 1. Induction of ATF2 translation by PTX and ADR in cancer cells. (A) Dose- and (B) time-dependent induction of ATF2 protein expression in Bel7402 cells treated with ADM and PTX. Bel7402 cells were treated with $0.4 \mu \mathrm{g} / \mathrm{ml}$ ADM and PTX for different times, or with ADM and PTX for $24 \mathrm{~h}$ at different doses, as indicated, and protein expression was examined by western blotting. (C) The messenger RNA expression levels of ATF2 upon treatment with or without increasing concentrations $(0.01,0.2$ and $0.4 \mu \mathrm{g} / \mathrm{ml})$ of ADM and PTX in Bel7402 cells were determined by reverse transcription-polymerase chain reaction. ATF2, activating transcription factor 2; ADR, adriamycin; PTX, paclitaxel.

at room temperature for $2 \mathrm{~h}$. Next, the membrane was incubated with the secondary antibody Goat Anti-Rabbit HRP (IgG H\&L) (1:3,000; ab6721; Abcam) at room temperature for 1.5-2.0 h. Finally, the antigen-antibody complexes were visualized by enhanced chemiluminescence (Thermo Fisher Scientific, Inc.).
Statistical analysis. Each experiment was performed $\geq 3$ times. All experimental data were analyzed using GraphPad Prism 5.0 (GraphPad Software, Inc., La Jolla, CA, USA) and presented as mean values \pm standard error of the mean. Statistical analysis was performed using the Student's t-test. $\mathrm{P}<0.05$ was considered to indicate a statistically significant difference. 
A

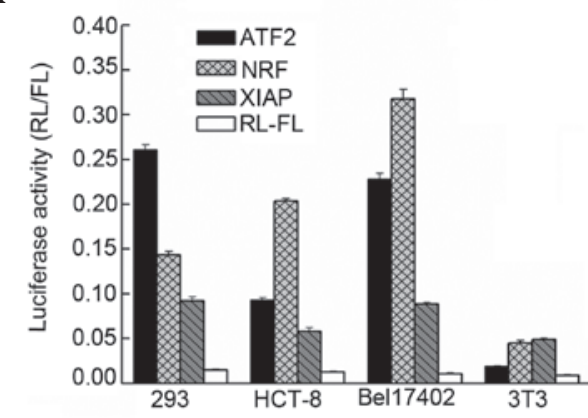

C

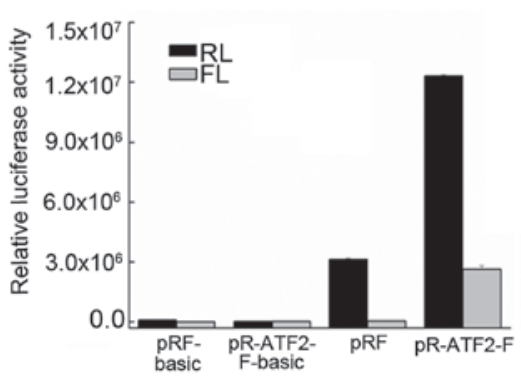

B

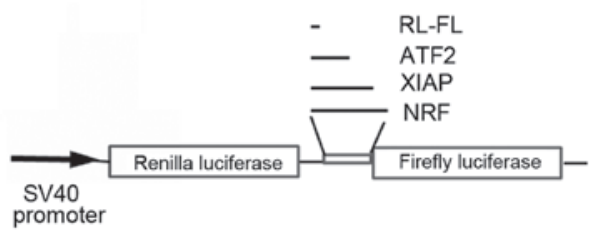

D

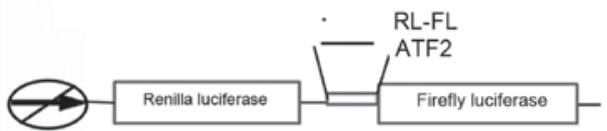

Figure 2. Identification of the IRES activity in the ATF2 5'-UTR. (A) The plasmids pRAF, pRXF, pRNF and pRF were transfected into the cell lines HEK293, Bel7402, HCT-8_and NIH-3T3. The IRES activity was expressed as the ratio of downstream cistron expression to upstream cistron expression (FL/RL). The error bars indicate the $\mathrm{SD}$ determined from $\geq 3$ independent experiments performed in triplicate. (B) Schematic representation of the expression cassette of the dual-luciferase bicistronic constructs pRF, pRAF, pRXF and pRNF. (C) The translation of the second cistron is not due to reinitiation or aberrant messenger RNA species. To test the cryptic promoter activity in the ATF2 5'-UTR, the sequence was cloned upstream of the FL reporter, and the SV40 promoter was removed. The empty vector was used as a negative control. The plasmids were transfected into Bel7402 cells. The RL and FL activities were measured after $24 \mathrm{~h}$. The error bars indicate the SD determined from $\geq 3$ independent experiments performed in triplicate. (D) Schematic representation of the expression cassette of the dual-luciferase bicistronic constructs $\mathrm{pRF}$ and $\mathrm{pRAF}$ in which the promoter was deleted. RL, Renilla luciferase; FL, firefly luciferase; SV, simian virus; ATF2, activating transcription factor 2; NRF, nuclear respiratory factor; XIAP, X-linked inhibitor of apoptosis protein; IRES, internal ribosome entry segment; UTR, untranslated region; SD, standard deviation.

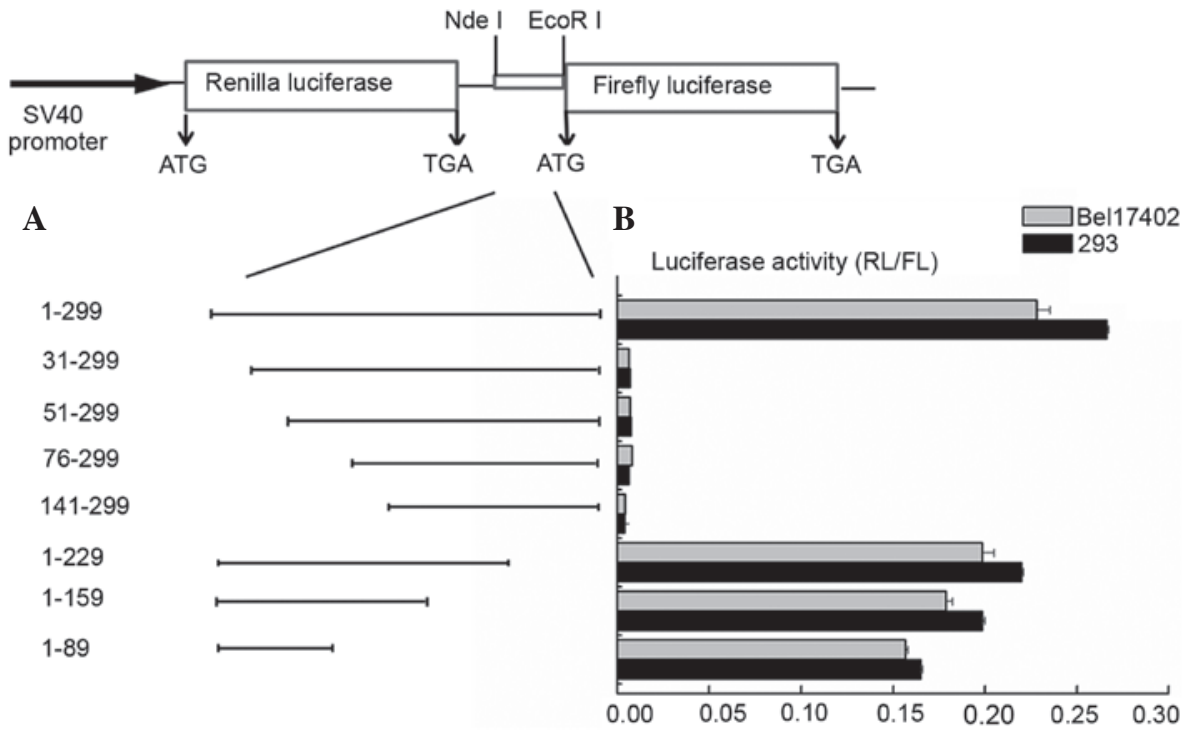

Figure 3. Mapping the ATF2 internal ribosome entry segment. (A) Schematic representation of bicistronic pRF constructs containing a series of 5'-3'- or 3'-5'-deleted ATF2 5'-untranslated region fragments. (B) HEK293 and Bel7402 cells were transfected with each indicated plasmid. After 24 h, the luciferase activities were tested. Data represent the mean \pm standard deviation of FL vs. RL activity in $\geq 3$ independent experiments. RL, Renilla luciferase; FL, firefly luciferase; SV, simian virus; ATF2, activating transcription factor 2

\section{Results}

Protein expression of ATF2 is induced at the translational level following treatment with chemotherapeutic drugs in Bel7402 cells. ATF2 exhibited very low expression in Bel7402 cells (Fig. 1A). Notably, when Bel7402 cells were treated with increasing concentrations $(0.01,0.2$ and $0.4 \mu \mathrm{g} / \mathrm{ml})$ of two chemotherapeutic drugs (ADM and PTX), ATF2 protein expression was induced following treatment with ADM and PTX (Fig. 1A) in a dose-dependent manner. To demonstrate 
A

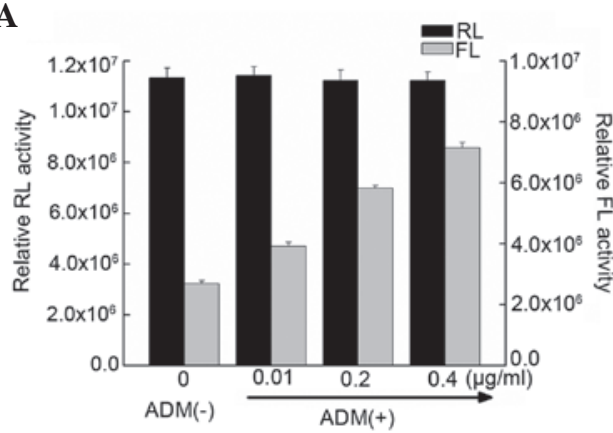

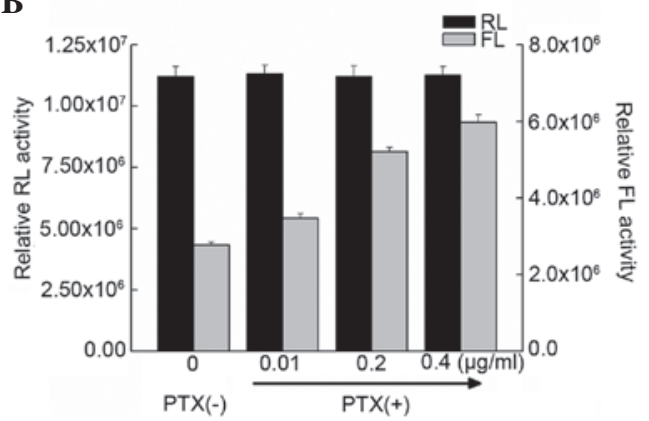

Figure 4. Transfection and reporter assay for the effect of ADM and PTX on ATF2 internal ribosome entry segment activity. (A and B) Bel7402 cells were transfected with the pRL-ATF2-FL plasmid and treated with or without increasing concentrations $(0.01,0.2$ and $0.4 \mu \mathrm{g} / \mathrm{ml})$ of $(\mathrm{A}) \mathrm{ADM}$ and $(\mathrm{B}) \mathrm{PTX}$. Data are represented as the mean \pm standard deviation of RL and FL activities in $\geq 3$ independent experiments. RL, Renilla luciferase; FL, firefly luciferase; ATF2, activating transcription factor 2; ADR, adriamycin; PTX, paclitaxel.

A

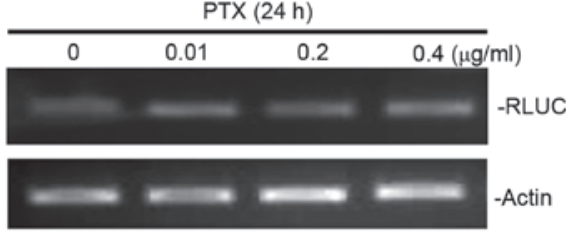

C

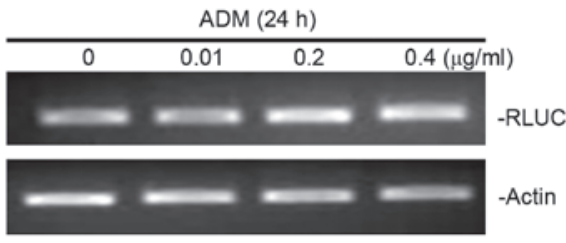

B

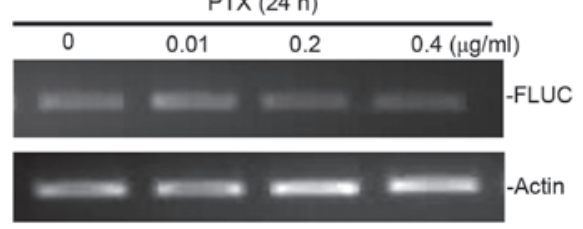

D

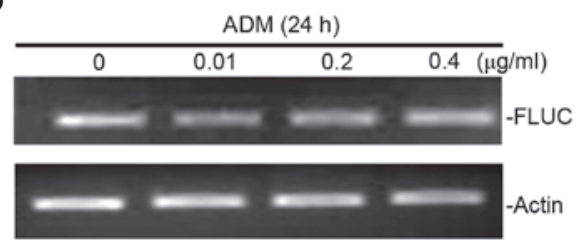

Figure 5. RLUC and FLUC mRNA expression levels are not affected by ADM or PTX treatment. (A and B) Bel7402 cells were transfected with the pRL-ATF2-FL plasmid and treated with or without increasing concentrations $(0.01,0.2$ and $0.4 \mu \mathrm{g} / \mathrm{ml})$ of PTX. The mRNA expression levels of (A) RLUC and (B) FLUC were determined by RT-PCR. (C and D) Bel7402 cells were transfected with the pRL-ATF2-FL plasmid and treated with or without increasing concentrations $(0.01,0.2$ and $0.4 \mu \mathrm{g} / \mathrm{ml})$ of ADM. The mRNA expression levels of (C) RLUC and (D) FLUC were determined by RT-PCR. RLUC, Renilla luciferase; FLUC, firefly luciferase; ATF2, activating transcription factor 2; ADR, adriamycin; PTX, paclitaxel; mRNA, messenger RNA; RT-PCR, reverse transcription-polymerase chain reaction.

whether the ATF2 induction changed with the exposure time to the drugs, ATF2 protein expression was detected at various times following $0.4-\mu \mathrm{g} / \mathrm{ml} \mathrm{ADM}$ and PTX treatment for 2, 4, 8,12 and $24 \mathrm{~h}$. Fig. 1B reveals that ATF2 protein expression is increased in a time-dependent manner following treatment with ADM and PTX. Next, the mechanism by which these drugs increased the levels of ATF2 in Bel7402 cells was examined. Treatment of Bel7402 cells with either ADM or PTX did not result in any significant change $(\mathrm{P}=0.552)$ in ATF2 mRNA levels (Fig. 1C), indicating that ADM/PTX-induced ATF2 expression occurs at the translational level.

ATF2 5'-UTR-mediated IRE in mammalian cultured cell lines. Since by searching the ATF2 gene in the human genome database (http://www.ncbi.nlm.nih.gov/nuccore/NM_001880.3) it was observed that the ATF2 5'-UTR is generally long (ATF2, $299 \mathrm{nt}$ ) and rich in GC, the possibility that the ATF2 5'-UTR contains an IRES that could regulate ATF2 protein synthesis following treatment with chemotherapeutic drugs was examined. To evaluate whether ATF2 translation was initiated from an IRE, the ATF2 5'-UTR was inserted between the two cistrons of a bicistronic vector, in which the first cistron encodes the RLUC gene and the second cistron encodes the
FLUC gene (Fig. 2A). The first cistron is proximal to the mRNA's cap structure, and therefore, is expected to be translated by the conventional cap-dependent translation mode. The second cistron is distal from the cap site; therefore, it is expected to undergo translation only upon insertion of an IRES element between the two cistrons (27). The bicistronic vectors pR-ATF2-F were transfected into HEK293 Bel7402 and HCT-8 human cells, and into murine NIH-3T3 cells. The pR-XIAP-F and pR-NRF-F vectors, which contain the well-characterized cellular XIAP and NRF IRES, were transfected as positive controls, while pRF was used as a negative control. The RL and FL activities were determined as aforementioned described. The IRES activity was calculated as the ratio of FLUC to RLUC normalized to that of pRF-transfected cells. As indicated in Fig. 2B, the ATF2 IRES was observed to be active in the HEK293, Bel7402 and HCT-8 cell lines, but not in the NIH-3T3 cell line. The ATF2 IRES activity was different in each cell line, being high in Bel7402 and HEK293 cells ( $\sim 5$-fold higher than the XIAP IRES activity). These results suggest that a putative IRES element was present in the 5'-UTR of the ATF2 mRNA. The difference in the levels of IRES activity observed in these cell lines is probably due to intrinsic differences between these cell types. 
Internal initiation of translation mediated by the ATF2 5'-UTR is not due to cryptic promoter activity. To exclude the possibility that the expression of the second cistron in these two bicistronic mRNAs could be derived from monocistronic RNAs due to the presence of a cryptic promoter in the 5'-UTR, pR-ATF2-F-basic plasmids were generated, in which the ATF2 5'-UTR was inserted in the promoterless pRF-basic vector upstream of the RLUC coding sequence. The HEK293 and Bel7402 cell lines were each transfected with the pRF plasmid (a vector containing the SV40 early promoter and serving as a positive control), the promoterless pRF-basic plasmid and the pR-ATF2-F-basic plasmid. Luciferase activities were measured as aforementioned described. Fig. 2C indicates that the FLUC activity measured in pR-ATF2-F-basic-transfected cells is comparable to that measured in cells transfected with the pRF-basic plasmid, and is $\sim 350$-fold lower than the activity produced by pR-ATF2-F. This result clearly illustrated that the ATF2 5'-UTR was devoid of promoter activity.

Mapping the ATF2 IRES. To further identify the core regions that promote the internal initiation of translation, serial truncations of the ATF2 5' leader sequence were created (Fig. 3A). Subsequently, the activities of the inserted sequences were assayed as aforementioned described. The ability of these truncated sequences to promote the translation of the mRNA was also compared with that of pRF. Deletions of $30 \mathrm{nt}$ of the 5' end of ATF2 resulted in a marked decrease in IRES activity, and further deletion of 52, 75 and 142 nt of the 5' end of ATF2 led to a similar reduction. Notably, at the $3^{\prime}$ end of ATF2, deletion of 70,140 and $210 \mathrm{nt}$ did not produce a significant decrease $(\mathrm{P}=0.148)$, since the IRES activity remained almost the same as that of the full-length ATF2 5'-UTR (Fig. 3B). These data indicated that the 5' end of ATF2 is important for positioning the IRES in context with the translational start site, and the core IRES region of ATF2 contains 30 bases and resided between $n t-299$ and -269 . The results were the same when different cells were transfected. These results demonstrated that the full length of the ATF2 5'-UTR contributes to its maximal IRES activity, and the 5' $30 \mathrm{nt}$ of the ATF2 5' leader region are essential for its IRES activity.

Induction of ATF2 IRES activity under cellular stress conditions. It has been reported that, for certain mRNAs, IRES-mediated initiation of protein translation is activated during conditions such as cellular stress and apoptosis (28). In the present study, ATF2 protein expression in Bel7402 cells was induced under treatment with ADM and PTX (Fig. 1). To investigate whether the ATF2 IRES activity is also affected by cellular stress, the ATF2 activity was examined in Bel7402 cells treated with two chemotherapeutic drugs (ADM and PTX), which induce a stress condition in cells. Gene transfections and reporter assays were performed to determine if ADM and PTX induced ATF2 IRES activity. Bel7402 cells were transfected with the pRF plasmid, containing the full-length fragment of the ATF2 5'-UTR sequence, and then treated with ADM and PTX. It was observed that the FLUC activity in transfected cells treated with ADM and PTX was higher than that of untreated cells (Fig. 4). Furthermore, RT-PCR analyses of mRNA expression of FLUC were performed in cells transfected with pRL-ATF2-FL following treatment with ADM and PTX. It was observed that FLUC mRNA expression was not altered by drugs-induced cellular stress (Fig. 5), suggesting that ADM and PTX induced ATF2 IRES activity.

\section{Discussion}

The transcription factor ATF2 is a nuclear target of stressactivated protein kinases such as p38, which are activated by various extracellular stresses $(29,30)$. Previous studies demonstrated that the stimulation of ATF2 mainly occurs by post-translational modification of protein subunits and changes in binding efficiency $(31,32)$. However, the regulation at the translational level of ATF2 is poorly understood. To the best of our knowledge, the present study provided the first evidence of IRES-mediated translation of the ATF2 5'-UTR by using bicistronic Renilla firefly luciferase assays. The present study has revealed that the ATF2 IRES has various IRES activities in different cell types. The differences in ATF2 IRES activity between the different cells lines examined in the current study may be due to the levels of expression of IRES-transactivating factors (ITAFs) required for its function, which are probably different in these cells (33).

By performing deletion mapping, the core IRES region of the ATF2 mRNA was identified to be located between nt -299 and -269 of the ATF2 5'-UTR. In certain cases, a specific and stable RNA structure is necessary for IRES activity (34). For example, a module with a 9-nt motif in the Gtx mRNA, a novel murine homeobox gene which in adult animals is specifically expressed within glial cells of the central nervous system, including the forebrain, and in germ cells of the testis (35), recruits the $40 \mathrm{~S}$ ribosomal subunit by base pairing to the $18 \mathrm{~S}$ ribosomal RNA (rRNA) and initiates the internal translation (36), and in c-Myc mRNA, there are two 14-nt segments that can recruit the $43 \mathrm{~S}$ preinitiation complex and activate the internal translation initiation (37). In line with these observations, our data lead to the postulation that the 30-nt short fragment of the ATF2 IRES is crucial for recruiting a ribosome and enhancing the initiation of translation, and this core IRES region of ATF2 may affect the translation efficiency through mRNA-rRNA interactions of these complementary regions. To gain more insight into the mechanism, further investigation is warranted to study the differences in the ability of recruiting a ribosome by the full-length ATF2 5'-UTR or the minimal ATF2 IRES region.

An important finding of the present study is that IRES-regulated ATF2 translation is activated upon the treatment of Bel7402 cells with two chemotherapeutic drugs, ADM and PTX. The difference in the ability to stimulate the expression of ATF2 protein may due to the treatment capability of these two drugs; perhaps the Bel7402 cells used in the present study have a greater ability to evade death during ADM therapy compared with PTX therpay. The expression and tissue distribution of ITAFs can change during cellular stress, and thus regulate IRES activity $(38,39)$. To understand the mechanism of initiation of translation of the ATF2 IRES, it will be necessary to perform further experiments in order to identify the ITAFs that regulate the IRES activity and to clarify the functional association between each of these proteins.

It has been reported that c-Jun/ATF2 heterodimers can mediate a response to certain stress signals (31). Transcriptional 
activation domains and the target genes of c-Jun/ATF2 heterodimers, which are implicated in growth control, include c-Jun itself, cyclin D1 and cyclin A (3). Notably, an IRES has been demonstrated to be involved in the translation of the mRNA of several ATF2 targets, including c-Jun and cyclin D1 $(40,41)$. This implies that IRES are very important in reposes to stress, and it would be relevant to investigate the ITAFs required for these IRES.

In the present study, it has been demonstrated that ATF2 5'-UTR harbors an IRES element localized between nt -299 and $n t-269$ upstream of the start codon, which would provide insights into the structure-function association of ATF2 in IRES-mediated translation. In addition, both ATF2 protein expression and IRES activity were increased in Bel7402 cells following treatment with the cellular stress-inducing chemotherapeutic drugs PTX and ADM, indicating the significant role of ATF2 IRES response to cellular stress in human hepatoma cells.

\section{Acknowledgements}

The present study was supported by the National Natural Science Foundation of China (Beijing, China; grant no. 81101667) and the Natural Science Foundation of Jiangsu Province (Nanjing, China; grant no. BK2009071).

\section{References}

1. Maekawa T, Sakura H, Kanei-Ishii C, Sudo T, Yoshimura T, Fujisawa J, Yoshida $\mathrm{M}$ and Ishii S: Leucine zipper structure of the protein CRE-BP1 binding to the cyclic AMP response element in brain. Embo J 8: 2023-2028, 1989.

2. van Dam H and Castellazzi M: Distinct roles of Jun: Fos and Jun: ATF dimers in oncogenesis. Oncogene 20: 2453-2464, 2001.

3. Maekawa T, Shinagawa T, Sano Y, Sakuma T, Nomura S, Nagasaki K, Miki Y, Saito-Ohara F, Inazawa J, Kohno T, et al: Reduced levels of ATF-2 predispose mice to mammary tumors. Mol Cell Biol 27: 1730-1744, 2007.

4. Bhoumik A and Ronai Z: ATF2: A transcription factor that elicits oncogenic or tumor suppressor activities. Cell Cycle 7: 2341-2345, 2008.

5. Yu T, Li YJ, Bian AH, Zuo HB, Zhu TW, Ji SX, Kong F, Yin de Q, Wang CB, Wang ZF, et al: The regulatory role of activating transcription factor 2 in inflammation. Mediators Inflamm 2014: 950472, 2014.

6. Morrison DK and Davis RJ: Regulation of MAP kinase signaling modules by scaffold proteins in mammals. Annu Rev Cell Dev Biol 19: 91-118, 2003.

7. van Dam H, Wilhelm D, Herr I, Steffen A, Herrlich P and Angel P: ATF-2 is preferentially activated by stress-activated protein kinases to mediate c-jun induction in response to genotoxic agents. Embo J 14: 1798-1811, 1995.

8. Papassava P, Gorgoulis VG, Papaevangeliou D, Vlahopoulos S, van Dam H and Zoumpourlis V: Overexpression of activating transcription factor-2 is required for tumor growth and progression in mouse skin tumors. Cancer Res 64: 8573-8584, 2004.

9. Bhoumik A, Huang TG, Ivanov V, Gangi L, Qiao RF, Woo SL, Chen SH and Ronai Z: An ATF2-derived peptide sensitizes melanomas to apoptosis and inhibits their growth and metastasis. J Clin Invest 110: 643-650, 2002.

10. Liu S, Wang F, Yan L, Zhang L, Song Y, Xi S, Jia J and Sun G: Oxidative stress and MAPK involved into ATF2 expression in immortalized human urothelial cells treated by arsenic. Arch Toxicol 87: 981-989, 2013.

11. Sheikh MS and Fornace AJ Jr: Regulation of translation initiation following stress. Oncogene 18: 6121-6128, 1999.

12. Lackner DH and Bähler J: Translational control of gene expression from transcripts to transcriptomes. Int Rev Cell Mol Biol 271: 199-251, 2008.
13. Shatsky IN, Dmitriev SE, Terenin IM and Andreev DE: Capand IRES-independent scanning mechanism of translation initiation as an alternative to the concept of cellular IRESs. Mol Cells 30: 285-293, 2010.

14. Stoneley M and Willis AE: Cellular internal ribosome entry segments: Structures, trans-acting factors and regulation of gene expression. Oncogene 23: 3200-3207, 2004.

15. Hellen CU and Sarnow P: Internal ribosome entry sites in eukaryotic mRNA molecules. Genes Dev 15: 1593-1612, 2001.

16. Komar AA and Hatzoglou M: Internal ribosome entry sites in cellular mRNAs: Mystery of their existence. J Biol Chem 280: 23425-23428, 2005.

17. Oumard A, Hennecke M, Hauser $H$ and Nourbakhsh M: Translation of NRF mRNA is mediated by highly efficient internal ribosome entry. Mol Cell Biol 20: 2755-2759, 2000.

18. Riley A, Jordan LE and Holcik M: Distinct 5' UTRs regulate XIAP expression under normal growth conditions and during cellular stress. Nucleic Acids Res 38: 4665-4674, 2010.

19. Shiroki K, Ohsawa C, Sugi N, Wakiyama M, Miura K, Watanabe M, Suzuki Y and Sugano S: Internal ribosome entry site-mediated translation of Smad5 in vivo: Requirement for a nuclear event. Nucleic Acids Res 30: 2851-2861, 2002.

20. Baird SD, Turcotte M, Korneluk RG and Holcik M: Searching for IRES. RNA 12: 1755-1785, 2006.

21. Nevins TA, Harder ZM, Korneluk RG and Holcik M: Distinct regulation of internal ribosome entry site-mediated translation following cellular stress is mediated by apoptotic fragments of eIF4G translation initiation factor family members eIF4GI and p97/DAP5/NAT1. J Biol Chem 278: 3572-3579, 2003.

22. Yang L, Gu L, Li Z and Zhou M: Translation of TRAF1 is regulated by IRES-dependent mechanism and stimulated by vincristine. Nucleic Acids Res 38: 4503-4513, 2010.

23. Chan CP, Kok KH, Tang HM, Wong CM and Jin DY: Internal ribosome entry site-mediated translational regulation of ATF4 splice variant in mammalian unfolded protein response. Biochim Biophys Acta 1833: 2165-2175, 2013.

24. Fabrizio C, Stefano M, Laura G, Marco N, Lorenzo M and Antonio O: Re: Baseline and early MR apparent diffusion coefficient quantification as a predictor of response of unresectable hepatocellular carcinoma to doxorubicin drug-eluting bead chemoembolization. J Vasc Interv Radiol 27: 1456-1458, 2016.

25. Chen L, Liu Y, Wang W and Liu K: Effect of integrin receptortargeted liposomal paclitaxel for hepatocellular carcinoma targeting and therapy. Oncol Lett 10: 77-84, 2015.

26. Xi G, Hu X, Wu B, Jiang H, Young CY, Pang Y and Yuan H: Autophagy inhibition promotes paclitaxel-induced apoptosis in cancer cells. Cancer Lett 307: 141-148, 2011.

27. Pelletier J and Sonenberg N: Internal initiation of translation of eukaryotic mRNA directed by a sequence derived from poliovirus RNA. Nature 334: 320-325, 1988.

28. Komar AA and Hatzoglou M: Cellular IRES-mediated translation: The war of ITAFs in pathophysiological states. Cell Cycle 10: 229-240. 2014.

29. Gozdecka M, Lyons S, Kondo S, Taylor J, Li Y, Walczynski J, Thiel G, Breitwieser W and Jones N: JNK suppresses tumor formation via a gene-expression program mediated by ATF2. Cell Rep 9: 1361-1374, 2014.

30. De Graeve F, Bahr A, Sabapathy KT, Hauss C, Wagner EF, Kedinger $\mathrm{C}$ and Chatton B: Role of the ATFa/JNK2 complex in Jun activation. Oncogene 18: 3491-3500, 1999.

31. Livingstone C, Patel G and Jones N: ATF-2 contains a phosphorylation-dependent transcriptional activation domain. EMBO J 14: 1785-1797, 1995.

32. Zeke A, Bastys T, Alexa A, Garai Á, Mészáros B, Kirsch K, Dosztányi Z, Kalinina OV and Reményi A: Systematic discovery of linear binding motifs targeting an ancient protein interaction surface on MAP kinases. Mol Syst Biol 11: 837, 2015.

33. King HA, Cobbold LC and Willis AE: The role of IRES trans-acting factors in regulating translation initiation. Biochem Soc Trans 38: 1581-1586, 2010.

34. Filbin ME and Kieft JS: Toward a structural understanding of IRES RNA function. Curr Opin Struct Biol 19: 267-276, 2009.

35. Komuro I, Schalling M, Jahn L, Bodmer R, Jenkins NA, Copeland NG and Izumo S: Gtx: A novel murine homeoboxcontaining gene, expressed specifically in glial cells of the brain and germ cells of testis, has a transcriptional repressor activity in vitro for a serum-inducible promoter. EMBO J 12: 1387-1401, 1993. 
36. Hu MC, Tranque P, Edelman GM and Mauro VP: rRNA-complementarity in the $5^{\prime}$ untranslated region of mRNA specifying the Gtx homeodomain protein: Evidence that base-pairing to 18S rRNA affects translational efficiency. Proc Natl Acad Sci USA 96: 1339-1344, 1999.

37. Cencig S, Nanbru C, Le SY, Gueydan C, Huez G and Kruys V: Mapping and characterization of the minimal internal ribosome entry segment in the human c-myc mRNA 5' untranslated region. Oncogene 23: 267-277, 2004.

38. Schepens B, Tinton SA, Bruynooghe Y, Beyaert R and Cornelis $\mathrm{S}$ : The polypyrimidine tract-binding protein stimulates HIF-1alpha IRES-mediated translation during hypoxia. Nucleic Acids Res 33: 6884-6894, 2005.

39. Petz M, Them N, Huber H, Beug H and Mikulits W: La enhances IRES-mediated translation of laminin B1 during malignant epithelial to mesenchymal transition. Nucleic Acids Res 40: 290-302, 2012
40. Blau L, Knirsh R, Ben-Dror I, Oren S, Kuphal S, Hau P, Proescholdt M, Bosserhoff AK and Vardimon L: Aberrant expression of c-Jun in glioblastoma by internal ribosome entry site (IRES)-mediated translational activation. Proc Natl Acad Sci USA 109: E2875-E2884, 2012.

41. Shi Y, Sharma A, Wu H, Lichtenstein A and Gera J: Cyclin D1 and c-myc internal ribosome entry site (IRES)-dependent translation is regulated by AKT activity and enhanced by rapamycin through a p38 MAPK- and ERK-dependent pathway. J Biol Chem 280: 10964-10973, 2005. 